\title{
Advances in Our Understanding of Acromegaly - Is There an Optimal Management Regimen?
}

\author{
Claire E Higham ${ }^{1}$ and Peter J Trainer ${ }^{2}$
}

1. Diabetes and Endocrinology Specialist Registrar; 2. Consultant Endocrinologist, Department of Endocrinology, Christie Hospital, Manchester

DOI:10.17925/EE.2009.05.00.51

\begin{abstract}
The two- to three-fold increased mortality associated with active acromegaly can be significantly decreased and even normalised with effective treatment to reduce growth hormone $(\mathrm{GH})$ and insulin-like growth factor-I (IGF-I) levels to within normal limits. This article addresses the advances in surgical techniques, new approaches in radiotherapy and the choice of medical treatments that make it possible to achieve biochemical remission and improve signs and symptoms of disease in almost every patient with acromegaly. The literature is reviewed with regard to the relative merits of each treatment method, recent controversies such as the role of radiotherapy and primary octreotide therapy are discussed and a treatment algorithm is proposed to provide a guide to management.
\end{abstract}

\section{Keywords}

Acromegaly, trans-sphenoidal surgery, radiotherapy, dopamine agonist, somatostatin analogue, pegvisomant, treatment algorithm

Disclosure: Claire E Higham has no conflicts of interest to declare. Peter J Trainer has received research support from Pfizer, Ipsen, Novartis and Novo Nordisk. Received: 6 May 2009 Accepted: 16 July 2009

Correspondence: Claire E Higham, Department of Endocrinology, Christie Hospital, Manchester, M20 4BX, UK. E: cehigham@doctors.org.uk

Active acromegaly is associated with a two- to three-fold increase in mortality that can be reduced to that of the background population with effective treatment to decrease growth hormone $(\mathrm{GH})$ and insulin-like growth factor-I (IGF-I) levels to within normal limits. Advances in surgical techniques, new approaches in radiotherapy and a choice of medical treatments make it possible to achieve biochemical remission in almost every patient with acromegaly. However, controversy persists around the optimal order and combination of modalities to achieve control of GH and IGF-I while at the same time relieving symptoms and preserving pituitary function. In particular, the place of surgery as first-line treatment has been challenged by increasing interest and evidence that primary medical therapy can bring disease control without risking hypopituitarism and the other complications of surgery.

\section{Surgery}

Even with the increasing popularity of primary medical therapy, the majority of patients with acromegaly will undergo trans-sphenoidal surgery (TSS) as it offers the prospect of rapid, cost-effective correction of tumour mass effect and elevated circulating GH levels. The results of surgery continue to improve as a consequence of innovations such as the endoscope and intra-operative imaging. In the most successful series, $70-80 \%{ }^{2,3}$ of patients achieve remission; however, the success of surgery is operator-dependent and unfortunately, as most patients are operated on by less experienced surgeons, the reality is not published by the leading centres. A recent analysis of the UK national acromegaly registry reported that, overall, $56 \%$ of patients with a microadenoma achieved remission (GH $<5 \mathrm{mU} / \mathrm{l})$; however, the rates varied between centres from 20 to $68 \%{ }^{4}$ As well as the compelling evidence that complication rates are lower with experienced surgeons, ${ }^{5}$ this means it is critical that optimal surgical management is performed in a dedicated regional centre with small numbers of surgeons operating on a sufficient caseload to maintain expertise. Other determinants of surgical outcomes are tumour characteristics such as size, local invasion and extrasellar extension. ${ }^{2}$ Large macroadenomas with extension into surrounding tissues are very unlikely to be amenable to surgical cure, and it has been argued that such patients would be better served by avoiding the risks of surgery, such as hypopituitarism, and receiving a somatostatin analogue (SSA) as first-line treatment. The combination of the recognition that some macroadenomas cannot be surgically cured and the variable standards of surgeons have been the major forces driving the interest in primary medical therapy with SSAS.

\section{Radiotherapy}

Radiotherapy treatment is effective at controlling $\mathrm{GH}$ secretion and tumour growth, with up to $80 \%$ of patients achieving a $\mathrm{GH}<5 \mathrm{mU} / \mathrm{l}$ at 15 years. ${ }^{6}$ Historically, in the UK fractionated three-field external-beam radiotherapy at a dose of 4,500cGy was used as second-line adjunctive therapy in patients with acromegaly not cured by surgery. However, it has fallen out of favour principally because of concerns about the consequences of hypopituitarism and evidence of an increased risk of a cerebrovascular accident (CVA). ${ }^{7}$ Stereotactic radiotherapy in the form of proton beam, Linac or Gamma Knife ${ }^{\odot}$ are developing as techniques for focusing high-dose radiation to well-defined residual tumour volume in a single session, while minimising scatter to the brain and surrounding tissues. Losa et al. reported that Gamma Knife radiotherapy given to 83 patients following debulking surgery resulted in an $85 \%$ remission rate at 10 years post-surgery, with only $10 \%$ of patients developing hypopituitarism. ${ }^{8}$ Further long-term safety data are required, but the method appears to be faster-acting at both lowering $\mathrm{GH}$ and causing hypopituitarism. 
Figure 1: Serum Insulin-like Growth Factor I Concentration in 19 Patients with Acromegaly

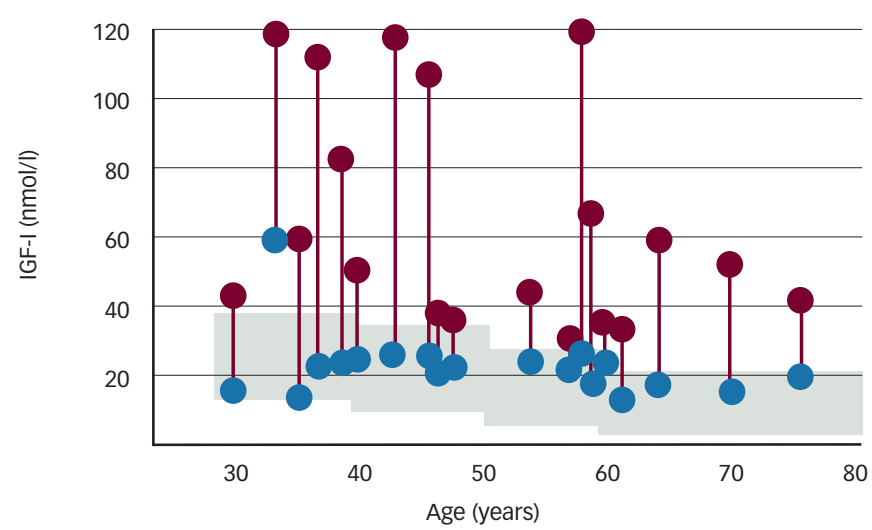

Pegvisomant has been used successfully on a weekly basis, albeit in combination with somatostatin analogues (SSAS). Nineteen patients with an insulin-like growth factor-I (IGF-I) above the upper limit of normal on maximum-dose SSA had pegvisomant added, initially $25 \mathrm{mg} /$ week up to $80 \mathrm{mg} /$ week. The addition of pegvisomant led to normalisation in 18 of 19 patients. However, this does not answer whether weekly administration is possible in patients on sole pegvisomant therapy. Serum IGF concentration in 19 patients with acromegaly before (represented by purple dots) and after (blue dots) six weeks of combined somatostatin analogue (SSA) and pegvisomant therapy. The shaded area represents ageadjusted IGF-I reference ranges.

Adapted from Feenstra et al., 2005.

In the absence of radiotherapy, there is no prospect of a patient being able to discontinue medical therapy; therefore, a financial case can be made for the continuing use of radiotherapy because with time patients may be able to stop high-cost medical treatment. That argument aside, as biochemical control can be achieved in almost every patient by a combination of surgery and medical therapy, we suggest the role of conventional radiotherapy in acromegaly is primarily for controlling continuing tumour growth. Stereotactic radiotherapy in its various forms is superseding multifractional radiotherapy and has a role in the treatment algorithm as an alternative to medical therapy in those patients who have a well-defined area of residual disease at least $5 \mathrm{~mm}$ away from the optic chiasm post-debulking surgery.

\section{Medical Therapy}

At some point in their care, the majority of patients with acromegaly will receive medical therapy, with the choice of agent and the timing of initiation being dependent on a number of factors, including tumour size and location, surgical and radiological intervention, patient choice, side effects and funding. Three classes of drug are available - SSAS, dopamine agonists (DAS) and the GH-receptor antagonist - with each having distinct advantages and drawbacks.

\section{Somatostatin Analogues}

Somatostatin is a widely distributed, naturally occurring 14 or 28 amino acid peptide that acts via a family of somatostatin receptors (SSTRS), of which there are five subtypes. The adult human pituitary expresses SSTR-1, $-2,-3$ and -5 subtypes, but the SSTR- 2 and to a lesser extent SSTR-5 are the principal receptors found on somatotroph adenomas, and their activation inhibits $\mathrm{GH}$ secretion. The short half-life of somatostatin (two to three minutes) means it is not a practical form of treatment. The SSAs octreotide and lanroetide are synthetic peptides with a longer half-life and greater receptor specificity for SSTR-2 and SSTR-5. SSAs are the first line of medical therapy following non-curative TSS; both octreotide and lanreotide are available as monthly depot formulations and are effective at achieving biochemical control and induce tumour shrinkage in $\leq 50 \%$ of patients (reviewed in reference 9).
It is difficult to judge the true efficacy of SSAs in achieving biochemical control post-surgery because of the varied entry criteria and therapeutic goals used by the plethora of publications that have addressed the topic. A meta-analysis that suggested that a long-acting repeatable (LAR) octreotide was superior to lanreotide at normalising IGF-I (63 versus $42 \%$ ) and reducing $\mathrm{GH}$ to $<2.5 \mathrm{ug} / \mathrm{l}(54 \text { versus } 48 \%)^{10}$ has been criticised, as $>50 \%$ of the patients included were from two studies that selected patients with proven octreotide responsiveness. Furthermore, the trials included were almost exclusively comparing lanreotide slow-release (SR) and not the more long-acting preparation, lanreotide Autogel ${ }^{\circledR}$. In reality, there is probably little difference in rates of biochemical control achievable with the two SSAs and the great majority of patients achieve symptomatic benefit, even if biochemical remission is achieved in approximately only $50 \%$ of 'unselected' patients. ${ }^{11}$ Lanreotide Autogel has the advantage that it can be self- or carer-administered. ${ }^{12}$

Correlations have been made between SSR expression by tumours and response to SSA, but the major predictors of the likelihood of biochemical control are pre-treatment GH and IGF-I levels, with more severe disease being less easily controlled.?

SSA are generally well-tolerated, although patients frequently experience abdominal symptoms on commencing the drugs; these usually spontaneously resolve. Deterioration in glucose tolerance due to inhibition of insulin secretion may result in diabetes. A further significant problem is that SSAs, by a combination of gallbladder stasis and alteration in the composition of bile, encourage gallstone formation. These are usually asymptomatic until SSAs are stoppe,d when restored gallbladder contractibility may precipitate gallstone-related problems. ${ }^{13}$

\section{Pre-operative Somatostatin Therapy}

SSAs have been used successfully for 20 years as medical therapy following non-curative surgery, but evidence is accumulating for their use as a primary treatment with the aim of achieving both biochemical control and tumour shrinkage without the associated risks of surgery or radiotherapy. Prospective studies of octreotide LAR in treatment-naïve patients with micro- or macroadenomas have demonstrated normalisation of GH or IGF-I levels in 25-70\% of patients in the first year, with rates improving with longer duration of therapy. A reduction in tumour size of at least $20 \%$ was seen in $75 \%$ of the patients, with a significant improvement in signs and symptoms of disease. ${ }^{14-17}$ It is unlikely that first-line SSA therapy will replace surgery in the majority of patients. However, the only study to randomise patients to either primary medical therapy with octreotide or surgery reported overal success rates at week 24 and 48 , respectively, of 25 and $28 \%$ for the octreotide LAR group and 49 and $39 \%$ for the surgery group..$^{18}$ Cure rates in both groups are disappointing and it is difficult to advocate a high-cost option that barely achieves disease control in one-quarter of patients. Patients with acromegaly rarely die from complications of tumour size; mortality relates to persistently raised GH and IGF-I levels. This leaves open the question as to what point in primary medical therapy patients with uncontrolled GH and IGF-I should proceed to surgery or alternative medications. A recent randomised, open-label, prospective trial comparing primary SSA therapy with surgery highlights this matter, with 13 out of 40 patients who were uncontrolled on octreotide LAR at 28 weeks opting for surgical intervention. ${ }^{18}$

The disappointing results of the only study to randomise patients to either primary medical therapy or surgery raises the question of whether SSAs are more likely to achieve biochemical control after non- 
curative debulking surgery. As already discussed, pre-treatment GH and IGF-I levels are the main determinant of the prospect of biochemical remission with an SSA. Petrossians et al. ${ }^{19}$ reported in a retrospective analysis of 24 patients that pre-operative SSA treatment achieved control of IGF-I in 29 and $45 \%$, respectively. After non-curative surgery, the control rates in the same patients rose to 54 and $78 \%$, respectively. Similar results have been reported by colao et al. ${ }^{20}$ and make a compelling argument that even if tumour size (or the choice of surgeon) makes the prospect of cure unlikely, the probability of subsequent biochemical remission with an SSA is improved by surgical debulking.

Another way of approaching primary SSA therapy is as a precursor rather than an alternative to surgery. The notion here is that SSA-induced tumour shrinkage may improve the prospect of surgical cure or reduce its complications. Active acromegaly causes hypertension, diabetes and reduced cardiac function, which increase surgical risk. It is also associated with hypertrophy of structures in the pharyngeal region, making intubation difficult. Although there are limited data in support, it seems likely that these factors increase surgical morbidity, which might be reversible with pre-operative medical therapy. Retrospective studies are conflicting, with some reporting no effect, ${ }^{21,22}$ while others report a modest benefit., ${ }^{23,24}$ The only prospective, randomised study demonstrated a modest but significant difference in IGF-I normalisation following surgery in patients with macroadenomas pre-treated with octreotide for six months compared with those not pre-treated (13/26 versus 4/25), although there was no difference if mean $\mathrm{GH}$ levels were used as criteria for cure. Preoperative SSA did not improve the complication rates and there was a suggestion that cure rates may actually be worse in patients with microadenomas..$^{25} \mathrm{An}$ interesting aside that probably reflects the reality of surgery for most patients, and not inconsistent with Colao et al., is that only $23 \%$ of patients were cured by surgery.

SSAs are safe, well-tolerated and the gold standard of post-surgical medical therapy. The plethora of studies addressing the use of preoperative or primary medical therapy suggest this may have a place in the acromegaly treatment algorithm, particularly in the short term, as an adjunct to surgery for macroadenomas or where there is limited surgical expertise. However, there are considerable cost implications for long-term primary medical therapy, and careful consideration is required in terms of intended duration of treatment.

\section{Dopamine Agonists}

DAs, of which cabergoline is the preferred agent, are relatively ineffective in achieving the modern standards of biochemical control for acromegaly, but have the virtues of being relatively inexpensive and oral. Cabergoline is not licensed for the treatment of acromegaly and systematic dose-finding studies have never been undertaken. The largest retrospective study demonstrated reductions in IGF-I and GH of $30-40 \%$, sufficient to normalise IGF-I and GH in around $30 \%$ of patients. ${ }^{26}$ They are particularly useful in patients with milder disease (serum IGF-I $<130 \%$ of the upper limit of normal) and those co-secreting prolactin ${ }^{27,28}$ or in combination with SSAs. ${ }^{29,30}$ Most clinicians do not exceed a dose of $1 \mathrm{mg}$ per day, although there are isolated reports of the successful use of higher doses. The future use of ergot-derived DAs, such as cabergoline, is in question because of concerns surrounding irreversible cardiac valve disease seen in patients with Parkinson's disease, who are treated with high doses up to six times the dose used for acromegaly. ${ }^{31,32}$ The mechanism is activation at the 5-HT2B receptor, as seen in the carcinoid syndrome. Patients treated with cabergoline require close monitoring by

\section{Figure 2: Treatment Algorithm for Acromegaly}

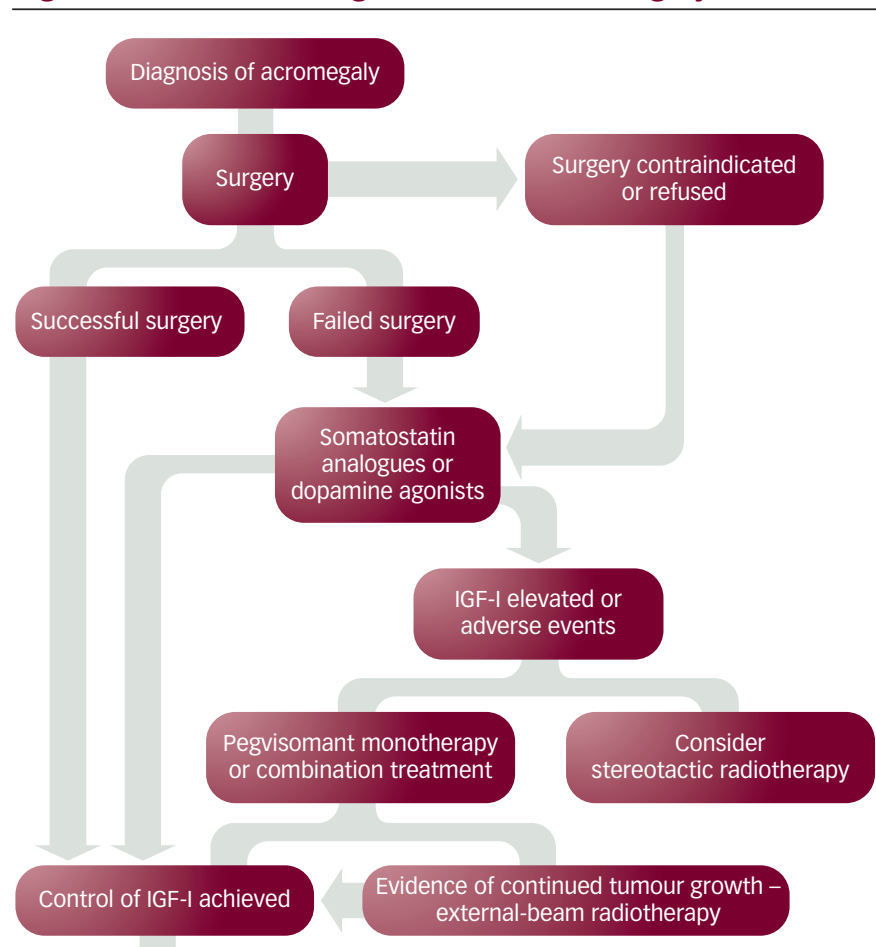

Monitor patient

If tumour growth is evident following initial surgery, consideration should be given to repeat surgery or conventional radiotherapy. IGF = insulin-like growth factor. Adapted from Clemmons et al., J Clin Endocrinol Metab, 2003;88:4759-67.

echocardiogram, and these concerns may lead to a reduction in cabergoline use despite the lack of evidence of increased risk at the doses used in acromegaly.

\section{Pegvisomant}

The GH-receptor antagonist pegvisomant is licensed in Europe for treatment of acromegaly in patients unresponsive to other forms of treatment. It is a pegylated $\mathrm{GH}$ analogue that acts as a competitive receptor antagonist at the $\mathrm{GH}$ receptor. As it does not lower serum $\mathrm{GH}$ levels, the primary marker of disease activity is serum IGF-I. Pegvisomant at doses $\leq 40 \mathrm{mg}$ per day can reduce IGF-I to within the reference range in $97 \%$ of patients with active, treated acromegaly, ${ }^{33}$ indicating that it should be possible with adequate dose titration to achieve a normal serum IGF-I in virtually every patient.

Although generally well-tolerated and effective at correcting the metabolic defects associated with acromegaly, careful monitoring of liver function is required as a significant rise in transaminases can occur, generally within the first month of therapy. The mechanism is poorly understood and idiosyncratic, and histologically has the appearance of drug-induced hepatitis. To date, it has resolved on stopping the drug, and in some cases even without discontinuation of the medication, with no patients having experienced long-term hepatic damage..$^{34,35}$ The nature of action of pegvisomant, in particular the stimulation of increased $\mathrm{GH}$ secretion, has resulted in an understandable concern that long-term therapy may be associated with tumour expansion. Reassuringly, the data from several sources suggest that the natural history of the tumours is unaltered by pegvisomant. Some tumours that were growing prior to pegvisomant have continued to expand, but there is no evidence of the antagonist inducing growth. ${ }^{34,36}$ 


\section{Combination Therapy}

Pegvisomant is indicated for patients unresponsive to SSA, and in that scenario the choice is whether to add it to ongoing SSA or substitute pegvisomant in place of SSA. Van der Lely's group have described the successful addition of weekly pegvisomant to monthly SSA. ${ }^{37,38}$ This is an attractive option as a means of maintaining any tumour shrinkage that has occurred with an SSA. The only reported study to randomise patients unresponsive to SSA to either combination therapy or pegvisomant monotherapy found similar rates of IGF-I normalisation in both groups, with patients on pegvisomant monotherapy requiring on average $5 \mathrm{mg}$ per day more than those on combination therapy ${ }^{39}$ - the implication being that there is little difference in the price of the two options, which is contrary to previous claims that combination treatment was significantly less expensive. The decision to use monotherapy or combination treatment depends on individual patient circumstances; for example, good tumour shrinkage with SSA would be a reason for combination treatment, while deteriorating glucose tolerance argues for monotherapy.

\section{Novel Treatments}

Pasireotide (SOM230) is a novel multireceptor ligand SSA with high binding affinity to SSTR-1, $-2,-3$ and -5 and up to 40 -fold greater affinity for SST5 than octreotide. It may have a role in the treatment of various types of pituitary tumour, including those secreting GH. A phase II study has demonstrated effective IGF-I and GH suppression with a significant reduction in tumour volume, but there are concerns in terms of deteriorating glucose tolerance. ${ }^{40}$ The future role of pasireotide in the treatment of acromegaly remains to be determined, but it will probably have a role in the treatment of octreotide-resistant tumours, particularly large adenomas. Another multireceptor ligand, the chimeric molecule BIM-23A760, that has activity at both somatostatin (SSTR 2 and 5) and dopamine receptors (DA2) is in clinical trials following encouraging in vitro results. ${ }^{41}$

\section{Optimal Management Regimen}

The treatment regimen for acromegaly should be individually tailored based on informed patient choice and the defined biochemical and clinical goals of treatment. Treatment decisions will take into consideration the desire to achieve biochemical control, relieve symptoms and signs, preserve pituitary function and control tumour size (see Figure 2).

We would advocate that first-line treatment for the great majority of patients remains TSS performed by an experienced surgeon. Microadenomas should be cured and debulking of macroadenomas will relieve pressure on structures such as the optic chiasm, and will also improve the likelihood of remission with secondary medical therapy.
Controversy remains in terms of the role of primary medical therapy with SSAs, particularly in centres without adequate neurosurgical expertise. We would certainly recommend pre-operative medical therapy in patients with complications such as sleep apnoea or cardiomyopathy. There is a lack of data to show that pre-treatment of macroadenomas improves surgical complications or remission rates, and we feel the currently available data in terms of long-term morbidity and mortality are insufficient to recommend it replace TSS, but it should be an option for patients who are not fit for surgery or under special circumstances.

Conventional radiotherapy should be reserved for patients with continued tumour growth despite surgical and medical therapy. However, stereotactic radiotherapy may have a specific role in those patients with a well-defined area of residual disease at least $5 \mathrm{~mm}$ away from the optic chiasm post-debulking surgery.

Despite these interventions, the majority of patients will require medical therapy. SSAs provide the mainstay of medical treatment, with DA being an option in those with mild elevations of IGF-I and co-secreting prolactin. In Europe, pegvisomant is limited to patients who remain uncontrolled on maximum-dose SSA or who are intolerant of SSAS due to side effects. Combination treatments of SSAS and pegvisomant are increasingly being used as there is some evidence of a synergistic effect of the two medications and benefit in those uncontrolled on either drug alone. The management of acromegaly is complex, but with improved surgical and radiotherapy techniques and multiple medical options, biochemical control should be achievable in all patients. As new medications and techniques evolve, the treatment paradigm will almost certainly change, and patients should be followed closely and re-evaluated on a regular basis.

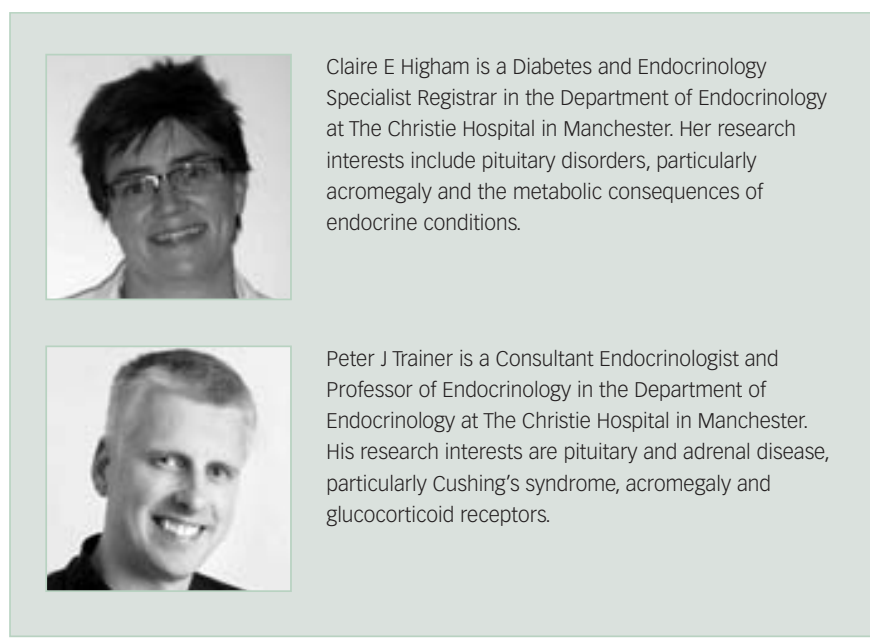

1. Holdaway IM, et al., J Clin Endocrinol Metab, 2004;89:667-74.

2. Abosch A, et al., J Clin Endocrinol Metab, 1998;83:3411-18.

3. Tindall GT, et al., J Neurosurg, 1993;78:205-15.

4. Bates PR, et al., Clin Endocrinol, 2008;68:136-42.

5. Ciric I, et al., Neurosurgery, 1997;40:225-36

6. Biermasz NR, et al., Clin Endocrinol (Oxf), 2005;3:321-7.

7. Ayuk J, et al., J Clin Endocrinol Metab, 2004;89:1613-17.

8. Losa M, et al., J Clin Endocrinol Metab, 2008;93:2546-52.

9. Bevan JS, J Clin Endocrinol Metab, 2005;90:1856-63.

10. Freda PU, et al., J Clin Endocrinol Metab, 2005;90:4465-73.

11. Murray RD, Melmed S, I Clin Endocrinol Metab, 2008;93:2957-68.

12. Bevan IS, et al., Clin Endocrinol (Oxf), 2008;68:343-9.

13. Paisley AN, et al., Clin Endocrinol (0xf), 2007;66:723-6.

14. Mercado M, et al., Clin Endocrinol (0xf), 2007;66:859-68.
15. Colao A, et al., Clin Endocrinol (0xf), 2006;64:342-51.

16. Cozzi R, et al., J Clin Endocrinol Metab, 2006;91:1397-1403.

17. Bevan JS, et al., J Clin Endocrinol Metab, 2002;87:4554-63.

18. Colao A, et al., Clin Endocrinol, 2009;70:757-68.

19. Petrossians $P$, et al., Eur J Endocrinol, 2005;152:61-6.

20. Colao A, et al., J Clin Endocrinol Metab, 2006;91:85-92.

21. Biermasz NR, et al., J Clin Endocrinol Metab, 1999;84:3551-5.

22. Abe T, Ludecke DK, Eur J Endocrinol, 2001;145:137-45.

23. Colao A, et al., Clin Endocrinol (Oxf), 2006;64:342-51.

24. Barkan AL, et al., J Clin Endocrinol Metab, 1988;67:1040-48.

25. Carlsen SM, et al., I Clin Endocrinol Metab, 2008;93:2984-90.

26. Abs R, et al., J Clin Endocrinol Metab 1998;83:374-6.

27. Colao A, et al., J Clin Endocrinol Metab, 1997;82:518-23.

28. Jackson SN, et al., Clin Endocrinol (0xf), 1997;46:745-9.

29. Cozzi R, et al., Clin Endocrinol (0xf), 2004;61:209-15.
30. Selvarajah D, et al., Eur J Endocrinol, 2005;152:569-74.

31. Schade R, et al., N Eng/ J Med, 2007;356:29-38.

32. Zanettini R, et al., N Eng/ J Med, 2007;356:39-46.

33. van der Lely AJ, et al., Lancet, 2001;358:1754-9.

34. Schreiber I, et al., Eur J Endocrinol, 2007;156:75-82.

35. Feenstra J, et al., Eur J Endocrinol, 2006;154:805-6.

36. Trainer PJ, et al., N Eng/ J Med, 2000;20:1171-7.

37. Feenstra J, et al., Lancet, 2005; 365:1644-6.

38. Neggers SJ, et al., J Clin Endocrinol Metab, 2007;92:4598-4601.

39. Trainer PJ, et al., Clin Endocrinol (Oxf), 2009 (Epub ahead of print).

40. Farrall AJ, et al., Pasireotide (SOM230) effectively reduces pituitary tumour volume in patients with active acromegaly: results from a phase II trial, OR53-4, proceedings of the 89th Annual Meeting of the Endocrine Society, 2007.

41. Jaquet $P$, et al., Eur J Endocrinol, 2005;153:135-41. 